\title{
Problematischer Alkohol-, Zigaretten-, Cannabis-, Glücksspiel- und Computerspielkonsum im Jugendal- ter: Sind Jungen gefährdeter?
}

Florian Rehbein, Eva-Maria-Zenses, Thomas Mößle

\section{Einleitung}

Weltweit stellt die menschliche Neigung zur Ausbildung süchtiger Verhaltensweisen ein gravierendes gesellschaftliches Problem dar. Unterschieden werden können hierbei Suchtformen, die sich auf Stoffe und Substanzen beziehen und solche, die sich auf exzessiv belohnende Verhaltensangebote beziehen. In Hinblick auf stoffgebundene Süchte wird für Deutschland aktuell davon ausgegangen, dass 9.5 Millionen Menschen einen riskanten Alkoholkonsum aufweisen, 1.4 Millionen Menschen medikamentenabhängig sind, 120,000 - 150,000 Menschen opiatabhängig sind und 600,000 Menschen einen gesundheitsschädlichen Cannabiskonsum aufweisen (Die Drogenbeauftragte der Bundesregierung, 2012). Als seit längerem klinisch anerkannte, wenngleich nosologisch als Impulskontrollstörung eingeordnete stoffungebundene Suchterkrankung, betrifft auch Glücksspielsucht eine Vielzahl von Menschen. Insgesamt weisen allein in Deutschland rund 275,000 Menschen ein problematisches und 264,000 Menschen ein pathologisches Glücksspielverhalten auf (Meyer, C. et al., 2011).

Süchte haben weitreichende Auswirkungen auf die Gesundheit indem sie das zentrale Nervensystem schädigen, das Risiko für andere Erkrankungen erhöhen, die Verkehrssicherheit beeinträchtigen und ein vermindertes psychosoziales Funktionsniveau, delinquente Handlungen sowie eine vorzeitige Sterblichkeit bedingen können (Anderson, Moller \& Galea, 2012; Baier \& Rabold, 2009; Pabst, Piontek, Kraus \& Müller, 2010). Nach der Weltgesundheitsorganisation stellen alleine die legalen Substanzen Alkohol und Tabak zwei der fünf wichtigsten gesundheitlichen Risikofaktoren dar, welche 4.4 Prozent bzw. 3.7 Prozent aller durch Krankheit verlorenen Lebensjahre verursachen (World Health Organization, 2009). Auch das pathologische Glücksspielverhalten als stoffungebundene Suchterkrankung führt in der Folge nahezu immer zu gravierenden finanziellen und psychosozialen Folgeschäden, die bis zum sozialen Abstieg, 
finanziellem Ruin und Straftaten führen können (Meyer, G. \& Bachmann, 2011).

\section{Das Jugendalter als Lebensphase besonderer Risikoneigung}

Im Jugendalter besteht ein besonderes Risiko für die Entwicklung missbräuchlicher und süchtiger Verhaltensweisen. Auf Basis einer deutschlandrepräsentativen Stichprobe von 44,610 Neuntklässlern ( $M=15.3$ Jahre) ermitteln Baier und Rabold (2009) für Alkohol ein Erstkonsumalter von 12.5 Jahren, für Zigaretten von 12.6 Jahren und für Cannabis von 14.1 Jahren. Bei dieser Studie ist jedoch zu berücksichtigen, dass ausschließlich Neuntklässler befragt wurden und damit Personen mit einem späteren Erstkonsum nicht in die Messung eingegangen sind. Es ist somit davon auszugehen, dass das tatsächliche Erstkonsumalter in der Studie leicht unterschätzt wird. Für das Glücksspielen ermittelt die Bundeszentrale für gesundheitliche Aufklärung (BZgA) ein Erstkonsumalter von rund 13.6 Jahren (Bundeszentrale für gesundheitliche Aufklärung, 2012). Anhand dieser Zahlen kann veranschaulicht werden, dass der durchschnittliche Erstkontakt zu den meisten Drogen und problematischen Verhaltensangeboten bereits im frühen Jugendalter stattfindet, womit der Umgang mit diesen Risikoeinflüssen eine besondere Entwicklungsaufgabe darstellt.

Doch wie kommt es dazu, dass Jugendliche bereits so früh mit diesen riskanten Angeboten in Berührung kommen? Riskante Verhaltensweisen können die Funktion haben, sich von den eigenen Eltern abzugrenzen und $\mathrm{zu}$ distanzieren, alltägliche Probleme und negative Stimmungen auszublenden, sich aber gleichsam als erwachsen und selbstbestimmt zu erleben (Baier \& Rabold, 2009; Pinquart \& Silbereisen, 2002; Rehbein, Kleimann \& Mößle, 2010). Weiterhin erleben Jugendliche oftmals, mit riskantem Verhalten auch gegenüber Gleichaltrigen „besonders gut anzukommen“: Man wirkt erwachsen, traut sich Dinge, die sich andere noch nicht zutrauen. Man entwickelt einen eigenen Lebensstil (dies wird auch in einigen Alkohol- oder auch Zigarettenwerbungen direkt aufgegriffen) und kann sich eventuell leichter dem anderen Geschlecht nähern (Pinquart \& Silbereisen, 2002). Da diese Motive jedoch nicht nur für das Jugendalter Gültigkeit beanspruchen können, stellt sich die Frage, was aus dem riskanten Probierverhalten im Übergang zum Erwachsenenalter wird. Hier wird häufig anhand einer drogenassoziierten Alterskurve argumentiert, dass sich die meisten riskanten und suchtähnlichen Verhaltensweisen mit dem Übergang in das Erwachsenenalter wieder normalisieren sollten (Muthén 
\& Muthén, 2000; Pinquart \& Silbereisen, 2002). Dies muss jedoch gerade für legale Drogen wie Alkohol oder Nikotin angezweifelt werden, da empirische Daten darauf hindeuten, dass einmal erworbene Verhaltensmuster bis ins Erwachsenenalter eine hohe Persistenz aufweisen (Baier, Rabold, Kappes \& Kudlacek, 2009). Auch aus der Konstanzer Adoleszenz-Studie ist bekannt, dass ca. 10 Prozent der Menschen, die in der Adoleszenz nicht rauchen aber rund 30 Prozent der Menschen, die in der Jugend zumindest gelegentlich rauchen, im Erwachsenenalter zu den häufigen Rauchern gehören (Fend, 2005). Zum Glücksspielverhalten liegen jedoch hiervon abweichende Daten vor. So konnte in einer Längsschnittstudie mit über 500 jugendlichen Australiern gezeigt werden, dass von den Jugendlichen, die Glücksspiele ausprobiert hatten, nur ein Viertel in jedem Folgejahr weiterspielte (Delfabbro, Winefield \& Anderson, 2009). Gerade ein kurzfristiges Probierverhalten muss im Jugendalter somit nicht eine problematische Entwicklung bis ins Erwachsenenalter vorhersagen.

\section{Besondere Gefährdung männlicher Jugendlicher}

Bereits im Jugendalter weisen männliche Personen ein besonderes Risiko für die Ausbildung problematischer Konsummuster auf. Baier und Rabold (2009) ermitteln auf Basis einer deutschlandrepräsentativen Befragung von 44,610 Neuntklässlern, dass sich Jungen signifikant häufiger in der Gruppe der problematischen Alkohol- (Jungen 30.3\%, Mädchen 15.3\%), Cannabis- (Jungen 5.6\%, Mädchen 2.5\%) und Konsumenten harter Drogen (Jungen 1.4\%, Mädchen 0.5\%) befinden. Beim Zigarettenkonsum finden sich hingegen keine statistisch bedeutsamen Geschlechtsunterschiede (Jungen 18\%, Mädchen 17.4\%). Übereinstimmend zu diesem Befund zeigt sich auch in den regelmäßig erfolgenden Datenerhebungen der Bundeszentrale für gesundheitliche Aufklärung, dass sich Jungen und Mädchen hinsichtlich ihres Tabakkonsums auch in den letzten 30 Jahren kaum unterschieden haben (vgl. Abbildung 1). Jungen wiesen jedoch durchgängig häufiger einen regelmäßigen Alkoholkonsum und seit 2001 auch einen höheren Cannabiskonsum auf als Mädchen. Seit 2004 ist die Entwicklung von Substanzkonsum im Jugendalter jedoch bei beiden Geschlechtern insgesamt rückläufig. 


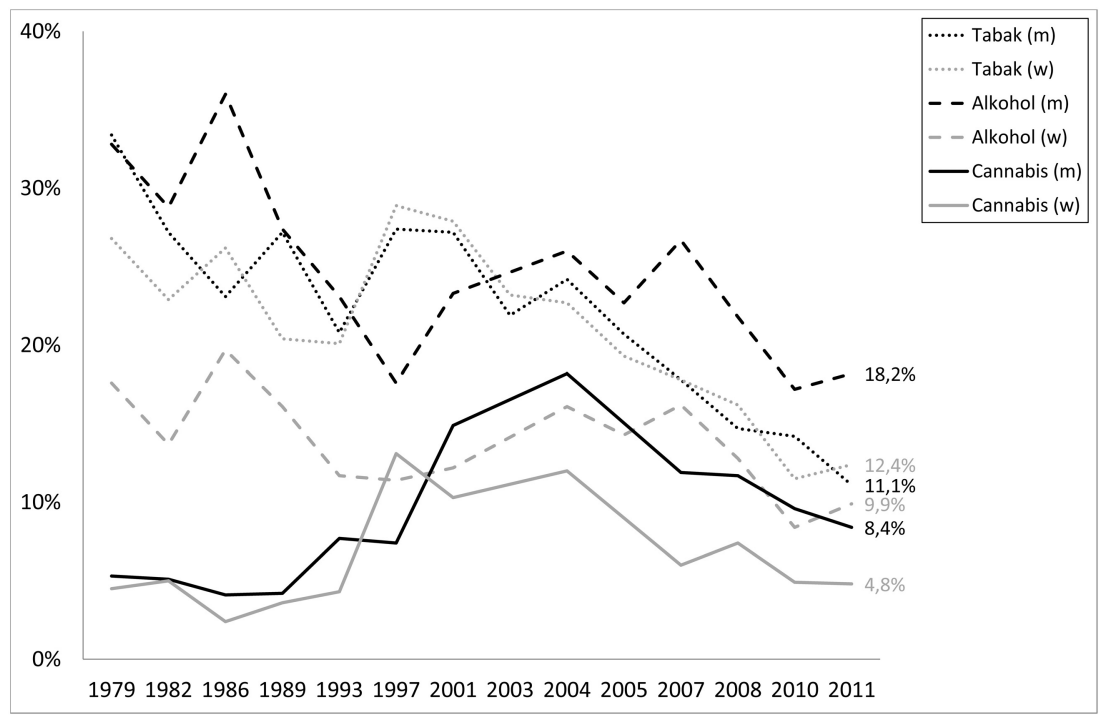

Abbildung 1. Anteil von Tabak-, Alkohol- und Cannabiskonsumenten ${ }^{1}$ unter männlichen und weiblichen Jugendlichen (12 - 17 Jahre) in Deutschland (Bundeszentrale für gesundheitliche Aufklärung, 2012).

Jungen sind gegenüber Mädchen nicht nur häufiger regelmäßige Konsumenten sondern weisen auch eine deutlichere Neigung zu problematischem und pathologischen Konsum auf. So konnte gezeigt werden, dass Jungen von Substanzmissbrauch und Substanzabhängigkeit 3- bis 6-mal häufiger betroffen sind als Mädchen (Ihle \& Esser, 2002). Ein pathologisches Glückspielverhalten im Alter von 16 bis 17 Jahren wiesen im Jahr 20110.5 Prozent der Jungen und 0.1 Prozent der Mädchen auf (Bundeszentrale für gesundheitliche Aufklärung, 2012). Auch in anderen Studien wurde gezeigt, dass männliche Jugendliche häufiger ein problematisches Glücksspielverhalten entwickeln als Mädchen (Schmidt, L. \& Kähnert, 2003). Bereits in den Jugenddaten bildet sich demnach das auch im Erwachsenenalter zu beobachtende erhöhte Risiko von männlichen Personen für die Ausbildung von Suchterkrankungen ab (Becker \& Hu, 2008). So konnte gezeigt werden, dass unter erwachsenen Hamburger Al-

1 Konsument nach BZGA-Definition: Alkoholkonsum (mindestens 1 mal wöchentlicher Konsum in den letzten 12 Monaten), Rauchen (ständiges oder gelegentlicher Konsum), Cannabis (Lebenszeitprävalenz) (Bundeszentrale für gesundheitliche Aufklärung, 2012). 
koholkonsumenten 31.2 Prozent der Männer und nur 14.7 Prozent der Frauen ein problematisches Trinkverhalten aufwiesen (Kraus, Pabst \& Müller, 2011). Aufgrund ihres erhöhten und häufiger problematisch betriebenen Alkoholkonsums weisen Männer auch eine erhöhte Rate an körperlichen Problemen auf, die durch Alkoholkonsum entstehen. So ist das Risiko aufgrund von Alkoholkonsum zu versterben bei Männern mittleren Alters in Europa besonders hoch (Anderson et al., 2012). Dies gilt jedoch auch für andere Drogen: Unter den 944 Menschen, die im Jahr 2012 in Deutschland an den Folgen ihres Drogenkonsums verstorben sind, betrug der Anteil männlicher Personen 81 Prozent.

\section{Computerspielsucht als ,neue“ stoffungebundene Suchterkrankung}

Im Folgenden soll mit der Computerspielsucht eine der vieldiskutierten neueren stoffungebundenen Süchte vorgestellt und intensiver beleuchtet werden. Diese wird auch in einer Darstellung der Ergebnisse einer aktuellen KFN-Studie in diesem Beitrag in den Vordergrund gestellt. Die Internet Gaming Disorder nimmt seit Mai 2013 den Status einer Forschungsdiagnose im DSM-5 ein (American Psychiatric Association, 2013). Durch die zunehmende Verbreitung von interaktiven Medien und der Nutzung des Internets wird seit den 90er-Jahren immer wieder über das mögliche Störungsbild Computerspielsucht diskutiert. Inzwischen konnte eine Vielzahl von Studien zeigen, dass gerade Nutzer von Videound Computerspielen einen psychopathologischen Symptomkomplex entwickeln können, der einige Ähnlichkeiten zu stoffgebundenen Süchten und dem pathologischen Glücksspiel aufweist. Hierbei stehen folgende Kriterien im Vordergrund: Kontrollverlust über das Spielverhalten, gedankliche und verhaltensbezogene Einengung auf das Spielverhalten und negative Konsequenzen, die zugunsten des Computerspielens in Kauf genommen werden. Außerdem werden Nervosität, Gereiztheit und Konzentrationsprobleme erlebt, wenn phasenweise nicht gespielt wird (Entzugserscheinungen) und die Spielzeit muss immer mehr gesteigert werden, um noch den gewünschten Effekt zu erzielen (Toleranzentwicklung).

\subsection{Fallbeispiel}

Die Problematik der Computerspielabhängigkeit soll im Folgenden anhand eines fiktiven Fallbeispiels verdeutlicht werden: Ein 21-jähriger Ma- 
schinenbaustudent schildert, dass er seit vielen Jahren exzessiv Computerspiele nutze. Zunächst einfache Jump-n-run Spiele, später vermehrt Rollenspiele und zwischendurch auch First-Person-Shooter. Seit ca. 2 Jahren spielt er League of Legends, zuvor hat er das Onlinerollenspiel World of Warcraft gespielt, welches ihm zunehmend langweilig wurde. Seine tägliche Spielzeit umfasst derzeit bis zu 10 Stunden, am Wochenende spiele er auch mal „durch“. Wenn er einmal begonnen habe zu spielen, könne er nicht mehr aufhören bis er ein bestimmtes Ziel erreicht habe, das Spielen dauere häufig länger als zuvor gedacht ("eben war es $20.00 \mathrm{Uhr}$ und dann ist es schon Mitternacht"). Er müsse auch ständig in der Uni über das Spielen nachdenken und wünsche sich dann lieber vor dem PC als dort zu sitzen. Seine Freundin beschwere sich häufig, dass er ihr nicht seine volle Aufmerksamkeit bieten könne, da er am Spielen sei. Er könne häufig abends nicht so gut einschlafen oder spiele so lange, dass seine Schlafzeit auf vier Stunden reduziert sei. Er habe häufig Augen- und Rückenschmerzen, spiele dann aber weiter und habe in den letzten Jahren durch sehr ungesundes Essen während des Spielens deutlich zugenommen. In der letzten Prüfungsphase seines Studiums habe er versucht das Spielen zu beenden, dieses habe zwei Tage geklappt, er sei dann unruhig geworden und gereizt, habe Streit mit seiner Freundin bekommen und sich nicht aufs Lernen konzentrieren können. In seinem Studium sei er so durchgekommen, die Bachelorarbeit bereite ihm aber Schwierigkeiten, da er häufig durchs Spielen abgelenkt sei und dann zu nichts komme.

\subsection{Verbreitung von Computerspielsucht}

0.9 bis 1.7 Prozent der Jugendlichen erfüllen nach repräsentativen deutschlandweiten Befragungen Kriterien einer Computerspielsucht und können als computerspielsüchtig eingeordnet werden (Rehbein et al., 2010; Schmidt, J.-H., Drosselmeier, Rohde \& Fritz, 2011). Bei den erwachsenen Computerspielern konnten Prävalenzzahlen zwischen 0.2 und 0.5 Prozent ermittelt werden (Festl, Scharkow \& Quandt, 2013; Schmidt, J.-H. et al., 2011). Wie bei den anderen diskutieren Suchtformen ergibt sich auf für die Computerspielsucht das Bild, dass Jungen weit häufiger davon betroffen sind als Mädchen. Nach der für die Neuntklässler repräsentativen Schülerbefragung des KFN sind 1.7 Prozent der Jungen aber nur 0.3 Prozent der Mädchen betroffen (Rehbein et al., 2010).

Empirische Daten deuten darauf hin, dass von Computerspielabhängigkeit betroffene Personen Auffälligkeiten aufweisen, die denen anderer 
Suchterkrankungen wie dem pathologischen Glücksspiel ähneln. Diese Auffälligkeiten sollen im Folgenden näher beschrieben werden.

\subsection{Psychische Auffälligkeiten computerspielsüchtiger Personen}

Insgesamt erscheinen auffällige Computerspieler gegenüber unauffälligen Spielern psychisch belasteter und weniger lebenszufrieden (Festl et al., 2013; Mentzoni et al., 2011; Starcevic, Berle, Porter \& Fenech, 2011). Unter Berufsschülern $(\mathrm{N}=2,667, \mathrm{M}$ [Alter] $=20$ Jahre $)$ empfinden sich computerspielsüchtige Personen aufgrund des Spielens in verschiedenen Lebensbereichen als beeinträchtigt (Rehbein, Mößle, Jukschat \& Zenses, 2011). Starke Beeinträchtigungen wurden in den Bereichen Freizeit (57\%), Soziales (41\%), Wohlbefinden (50\%), Familie (34\%) und Leistungsfähigkeit (32\%) berichtet. In einer aktuellen Schülerbefragung mit 4,436 Jugendlichen (7. bis 10. Jahrgänge) berichten 43 Prozent der computerspielabhängigen Jugendlichen einen leichten, 31 Prozent einen deutlichen und 14 Prozent sogar einen massiven Leidensdruck (Rehbein \& Mößle, 2013). Selbst unter Inanspruchnehmern einer Spielambulanz $(\mathrm{N}=131)$ weisen als computerspielabhängig klassifizierte Personen eine höhere psychische Belastung auf als Personen, die nach Eingangsdiagnostik als unauffällig eingeordnet werden (Beutel, Hoch, Wölfling \& Müller, 2011). Auch die Selbsteinschätzung von jugendlichen Computerspielsüchtigen zeigt, dass diese sich häufig auch nach eigener Einschätzung als computerspielsüchtig empfinden (Rehbein et al., 2010; Rehbein \& Mößle, 2013; Gentile, 2009). Besonders besorgniserregend erscheint, dass computerspielabhängige Jugendliche häufiger Suizidgedanken haben. Während nur rund 3 Prozent der unauffälligen Jugendlichen akute Suizidgedanken aufweisen trifft dies auf rund 13 Prozent der computerspielabhängigen Jungen und über 40 Prozent der computerspielabhängigen Mädchen zu (Rehbein \& Mößle, 2013). Im Bereich psychischer Komorbiditäten scheinen depressive Störungen, das Aufmerksamkeitsdefizit- und Hyperaktivitätssyndrom (ADHS) und Ängste häufiger vorzuliegen (Beutel et al., 2011; Mentzoni et al., 2011; Bioulac, Arfi \& Bouvard, 2008; Gentile et al., 2011). 


\subsection{Soziale Auffälligkeiten computerspielsüchtiger Personen}

Auch in sozialen Beziehungen berichten Computerspielabhängige häufiger Konflikte (Batthyány, Müller, Benker \& Wölfling, 2009) und erleben zudem in geringerem Maße soziale Unterstützung (Lee \& Kwon, 2001; Mößle \& Rehbein, 2013). Hierbei scheinen die betroffenen Personen im Bereich Aggressivität selber erhöhte Werte aufzuweisen (Lee \& Kwon, 2001). Aggressive Durchbrüche können insbesondere bei Störungen des Spielens auftreten: So gaben rund 30 Prozent der computerspielsüchtigen Jungen an, schon einmal eine andere Person in diesem Kontext körperlich angegriffen oder verletzt zu haben, wohingegen unauffällige Jungen dies kaum berichteten (Rehbein \& Mößle, 2013). Diese erhöhte Aggressionsneigung konnte bei computerspielabhängigen Mädchen hingegen nicht aufgefunden werden (Rehbein \& Mößle, 2013).

Auch das Freizeitverhalten scheint bei computerspielabhängigen Personen stärker eingeengt und die Teilnahme an organisierten Freizeitaktivitäten verringert zu sein (Achab et al., 2011; Rehbein et al., 2010). Computerspielabhängige weisen zudem in Hinblick auf ihre Leistungsfähigkeit unterschiedliche Auffälligkeiten wie etwa ein geringeres schulbezogenes Funktionsniveau auf (Rehbein \& Mößle, 2013; Mößle \& Rehbein, 2013). Zudem berichten betroffene Jugendliche davon, sich schlechter auf den Unterricht konzentrieren zu können (Gentile, 2009; Batthyány et al., 2009). Die Wahrnehmung der Schule ist ebenfalls verändert, indem positiven Aussagen zur Schule seltener zugestimmt wird (Mößle, 2012) und der Schule häufiger mit Ängstlichkeit begegnet wird (Batthyány et al., 2009; Young, 2010).

Auch ein häufigeres Schulschwänzen computerspielabhängiger Jugendlicher wurde mehrfach berichtet (Batthyány et al., 2009; Rehbein, Kleimann \& Mößle, 2009; Rehbein et al., 2010; Young, 2010), konnte in einer aktuellen Studie der Autoren jedoch nicht repliziert werden (Rehbein \& Mößle, 2013). Die Noten erscheinen jedoch in jedem Fall auffällig: So konnte belegt werden das computerspielabhängige Jugendliche signifikant schlechtere Noten in den Fächern Mathematik, Deutsch, Geschichte und Sport und auch im generellen Notendurchschnitt aufwiesen (Rehbein et al., 2010; Rehbein \& Mößle, 2013). Schlechtere Schulnoten scheinen längsschnittlich gesehen eher eine Folge als eine Ursache von Computerspielabhängigkeit zu sein (Gentile et al., 2011). Es bestehen jedoch auch Hinweise darauf, dass sich ein schulischer Leistungsabfall und die Verfestigung einer Computerspielabhängigkeit wechselseitig beeinflussen können (Mößle, 2012). 


\subsection{Psychosomatische Auffälligkeiten computerspielsüchtiger Personen}

In Hinblick auf Maße gesundheitlicher Beeinträchtigung zeigt sich eine verringerte Schlafzeit computerspielabhängiger Personen (Achab et al., 2011; Rehbein \& Mößle, 2013). So schlafen computerspielabhängige Jungen durchschnittlich 70 Minuten weniger als unauffällige Jungen (Rehbein \& Mößle, 2013). Zudem berichtet ungefähr jeder fünfte computerspielabhängige Jugendliche über erhebliche Einschlafschwierigkeiten (Rehbein \& Mößle, 2013). Ein verstärktes Vorliegen psychosomatischer Beeinträchtigungen (Empfinden von Kopf- oder Bauchschmerzen) konnte bei computerspielabhängigen Jugendlichen ebenfalls nachgewiesen werden (Batthyány et al., 2009).

\section{Computerspielsüchtige und glücksspielsüchtige Jugendliche im Ver-} gleich: Erste Ergebnisse der Studie Adolescent Development of Internet Gaming Disorder (ADIGD)

Die bisherigen Ausführungen haben verdeutlicht, dass männliche Personen besonders gefährdet sind eine Suchterkrankung zu entwickeln. Diesbezüglich stellt das frühe Jugendalter einen besonders kritischen Lebensabschnitt dar, da hier der Erstkontakt mit den meisten Suchtmitteln und problematischen Verhaltensangeboten erfolgt und dem jugendlichen Konsumverhalten ein bedeutsamer Einfluss auf die Entwicklung eines problematischen und süchtigen Konsumverhaltens im Erwachsenenalter zugesprochen wird. Es konnte zudem gezeigt werden, dass die Studienlage bereits auf einige Ähnlichkeiten zwischen stoffgebundenen und stoffungebundenen Süchten hinweist. Jedoch liegen bislang kaum Studien vor, die übergreifend klassisch stoffgebundene und stoffungebundene Suchterkrankungen beschreiben und im Rahmen vergleichender Auswertungen auch die Computerspielsucht berücksichtigen.

In diesem Abschnitt sollen Daten zur problematischen Nutzung von Drogen (Alkohol, Zigaretten, Cannabis) und potentiell psychotropen Verhaltensangeboten (Glücksspiele, Computerspiele) im Jugendalter berichtet werden. Datengrundlage ist der erste Messzeitpunkt der Adoleszent Development of Internet Gaming Disorder (ADIGD) mit $\mathrm{N}=4,436$ Jugendlichen, der im Herbst 2011 in 10 Schulen im Raum Hannover durchgeführt wurde (zur Methodik vgl. Rehbein \& Mößle, 2013). Die Studie wurde als Vollerhebung aller siebten bis zehnten Jahrgänge an zehn Schulen durchgeführt, die so ausgewählt wurden, dass sie sich möglichst gleichmäßig 
auf das Stadtgebiet und die ländliche Region von Hannover verteilen (zwei Gesamtschulen, vier Realschulen, vier Gymnasien). Da die Stichprobe trotz ihrer Größe keine bundesweite oder bundeslandweite Repräsentativität für sich beanspruchen kann, wird in den nachfolgenden Auswertungen ein inhaltlicher Schwerpunkt auf vergleichenden Analysen zwischen soziodemographischen Sub- und Belastungsgruppen innerhalb der Stichprobe gelegt werden.

Folgende Fragestellungen sollen im Rahmen einer explorativen Datenanalyse beantwortet werden:

1. In welcher Weise unterscheiden sich Jungen und Mädchen hinsichtlich der Nutzung psychotroper Substanzen und Verhaltensangebote?

2. Weisen Jungen häufiger als Mädchen eine psychische Abhängigkeit von Glücks- und Computerspielen auf?

3. Wie belastet sind Jungen, die eine Computerspielsucht oder Glücksspielsucht aufweisen im Vergleich zu unauffälligen Jungen?

4. Inwieweit bestehen Unterschiede in der Belastung zwischen computerspielsüchtigen und glücksspielsüchtigen Jungen?

Um ein allgemeines Bild von der Nutzung problematischer Substanzen und Verhaltensangebote im Jugendalter zu gewinnen, wurden die Jugendlichen zunächst zur Häufigkeit des Konsums von alkoholischen Getränken, Zigaretten, Cannabis, Glücksspielen und Computerspielen in den letzten 12 Monaten befragt (7-stufig: nie, 1- oder 2-mal, 3 bis 12-mal, mehrmals pro Monat, einmal pro Woche, mehrmals pro Woche, täglich) ${ }^{2}$. Für jede Substanz wurden Schwellenwerte der problematischen Nutzung nach Baier und Rabold (2009) zugrunde gelegt. Hinsichtlich Alkohol definieren die Autoren einen Konsum von mindestens einmal wöchentlich als

2 Bei der Abfrage der Konsumhäufigkeit von Alkohol wurden verschiedene Getränkesorten in einer einzelnen Frage zusammengefasst (Bier, Wein, Alcopops, Schnaps). Bei der Abfrage der Konsumhäufigkeit von Glücksspielen wurden hingegen fünf verschieden Glücksspielkategorien getrennt erhoben (Privates Glücksspiel um echtes Geld, Glücksspiele im Internet, Geldspielautomaten, Rubbellose, Sportwetten) und dann das individuell am häufigste genutzte Glücksspiel zugrunde gelegt (Max-Kodierung). In gleicher Weise wurde bei der Abfrage der Konsumhäufigkeit von Computerspielen verfahren, für die getrennt das Spielen auf zwei verschiedenen Plattformen zugrunde gelegt wurden (Spiele auf PC oder Mac, Spiele auf Spielkonsolen). 
problematisch, für Zigaretten einen täglichen Konsum und für Cannabis einen mindestens mehrmaligen Konsum pro Monat. In Anlehnung an diese Klassifikation wird in der vorliegenden Studie für das Glücksspielen ebenfalls ein mindestens mehrmaliger Konsum pro Monat und für das Computerspielen ein täglicher Konsum als problematischer Konsum definiert.

Es zeigt sich, dass Jungen signifikant häufiger als Mädchen einen problematischen Alkohol-, Cannabis-, Glücksspiel- und Computerspielkonsum aufweisen (vgl. Tabelle 1). Dies gilt jedoch nicht für den problematischen Zigarettenkonsum, der erwartungsgemäß nicht zwischen den Geschlechtern variiert und den rund 5.4 Prozent der Jungen und Mädchen aufweisen. Die größten Geschlechtsunterschiede werden bezüglich des problematischen Konsums der potentiell psychotropen Verhaltensangebote Glücksspiele und Computerspiele aufgefunden.

Für die problematische Nutzung von Substanzen wird ein signifikanter Anstieg von der 7. bis 10. Klasse erkennbar. Ein solcher Anstieg ist hingegen für die problematische Nutzung von Glücksspielen nicht und für die problematische Nutzung von Computerspielen nur eingeschränkt nachweisbar. Über alle Substanzen und Verhaltensangebote hinweg zeigt sich jedoch, dass diese von den Realschülern der Stichprobe am häufigsten und von den Gymnasiasten der Stichprobe am seltensten in problematischer Weise genutzt werden. 
Tabelle 1. Anteil von Jugendlichen mit problematischer Konsummenge von Drogen und potentiell psychotropen Verhaltensangeboten.

\begin{tabular}{|c|c|c|c|c|c|}
\hline & $\begin{array}{c}\begin{array}{c}\text { Alkohol- } \\
\text { konsum } \\
\text { (mind. }\end{array} \\
\text { einmal pro } \\
\text { Woche) } \\
\end{array}$ & $\begin{array}{c}\text { Zigaret- } \\
\text { ten- } \\
\text { konsum } \\
\text { (täglich) }\end{array}$ & $\begin{array}{c}\text { Cannabis- } \\
\text { konsum } \\
\text { (mind. mehr- } \\
\text { mals pro Mo- } \\
\text { nat) }\end{array}$ & $\begin{array}{c}\text { Glücksspiel- } \\
\text { konsum } \\
\text { (mind. mehr- } \\
\text { mals pro Mo- } \\
\text { nat) } \\
\end{array}$ & $\begin{array}{l}\text { Computerspiel- } \\
\text { konsum } \\
\text { (täglich) }\end{array}$ \\
\hline $\begin{array}{l}\text { Jungen } \\
(n=2,129)\end{array}$ & $7.1 \%$ & $5.4 \%$ & $2.4 \%$ & $4.6 \%$ & $30.2 \%$ \\
\hline $\begin{array}{l}\text { Mädchen } \\
(n=2,271)\end{array}$ & $4.9 \%$ & $5.4 \%$ & $1.3 \%$ & $0.6 \%$ & $2.5 \%$ \\
\hline Cramers V & $.047 * *$ & $.001^{\mathrm{ns}}$ & $.040 * *$ & $.126^{* * *}$ & $.378 * * *$ \\
\hline $\begin{array}{l}\text { 7. Klasse } \\
(n=1,072)\end{array}$ & $0.8 \%$ & $0.8 \%$ & $0.3 \%$ & $2.2 \%$ & $13.5 \%$ \\
\hline $\begin{array}{l}\text { 8. Klasse } \\
(n=1,176)\end{array}$ & $1.7 \%$ & $2.3 \%$ & $1.0 \%$ & $2.2 \%$ & $15.3 \%$ \\
\hline $\begin{array}{l}\text { 9. Klasse } \\
(n=1,198)\end{array}$ & $7.2 \%$ & $9.2 \%$ & $2.3 \%$ & $2.4 \%$ & $17.4 \%$ \\
\hline $\begin{array}{l}\text { 10. Klasse } \\
(n=990)\end{array}$ & $15.2 \%$ & $9.4 \%$ & $3.7 \%$ & $3.5 \%$ & $17.3 \%$ \\
\hline Cramers V & $.234 * * *$ & $.172 * * *$ & $.094 * * *$ & $.033^{\mathrm{ns}}$ & $.044 *$ \\
\hline $\begin{array}{l}\text { Realschulen } \\
(\mathrm{n}=\mathbf{1 , 2 8 6})\end{array}$ & $7.9 \%$ & $9.7 \%$ & $2.7 \%$ & $3.9 \%$ & $20.2 \%$ \\
\hline $\begin{array}{l}\text { Gymnasium } \\
(\mathrm{n}=\mathbf{1 , 8 1 5})\end{array}$ & $4.0 \%$ & $1.3 \%$ & $1.1 \%$ & $1.7 \%$ & $12.4 \%$ \\
\hline $\begin{array}{l}\text { Gesamtschule } \\
(n=1,335)\end{array}$ & $7.0 \%$ & $6.9 \%$ & $1.9 \%$ & $2.4 \%$ & $16.5 \%$ \\
\hline Cramers V & $.073 * * *$ & $.159 * * *$ & $.052 * *$ & $.058 * * *$ & $.088 * * *$ \\
\hline
\end{tabular}

Anmerkung. $* p<.05 ; * * p<.01 ; * * * p<.001$; ns $=$ nicht signifikant.

Weiterhin sollte eine psychisch abhängige Nutzung von Computer- und Glücksspielen erfasst werden. Zur verdachtsdiagnostischen Abklärung von Computerspielsucht wurde die Computerspielabhängigkeitsskala CSAS-II eingesetzt (Rehbein et al., 2009, 2010). Die Skala besteht aus 14 Items (4stufige Skala: 1, stimmt nicht bis 4, stimmt genau) und erfasst die Kriterien Einengung des Denkens und Verhaltens, Fortsetzung trotz negativer Konsequenzen, Kontrollverlust, Entzugserscheinungen und Toleranzentwicklung (Range: $14-56 ; \alpha=.93$ ). Als pathologisch gelten Spieler, die mindestens 42 Punkte erreichen, womit im Mittel allen Items zugestimmt 
wird. Das Instrument wurde nur Jugendlichen vorgelegt, die in den letzten 12 Monaten mindestens ein- oder zweimal ein Computerspiel am PC, an der Spielkonsole, am Handy oder an einer mobilen Spielkonsole gespielt haben.

Für die Erfassung pathologischen Glücksspielens wurde der Glücksspiel-Screen DSM-IV-MR-J (Fisher, 1999, 2000) nach deutscher Adaption von Schmidt und Kähnert (2003) eingesetzt. Das Instrument erfasst die neun DSM-IV Kriterien Starke Eingenommenheit, Toleranzentwicklung, Kontrollverlust, Entzugserscheinungen, Flucht, dem Verlust „hinterherjagen“, Lügen, illegale/antisoziale Handlungen, Gefährdung von Beziehungen und Ausbildung (Range: $0-9 ; \alpha=.78$ ). Die Fragen beziehen sich dabei gemäß DSM-IV auf das Vorliegen von Symptomen in den letzten 12 Monaten. Als pathologisch gelten Spieler, die vier oder mehr der erfragten Kriterien erfüllen. Das Instrument wurde nur Jugendlichen vorgelegt, die in den letzten 12 Monaten mindestens ein- oder zweimal an mindestens einem der fünf abgefragten Glücksspielformen teilgenommen haben.

Insgesamt weisen 52 Jugendliche (1.3\%) eine Computerspielabhängigkeit nach CSAS-II und 33 Jugendliche $(0.8 \%)$ ein pathologisches Glücksspielverhalten nach Glücksspiel Screen auf (vgl. Abbildung 2). Wie sich zeigt sind die Jungen von beiden stoffungebundenen Süchten weit stärker betroffen als die Mädchen. Die Geschlechterrelationen fallen zwischen beiden Störungen insgesamt ähnlich aus, wobei Mädchen unter den glücksspielsüchtigen Jugendlichen proportional einen etwas höheren Anteil ausmachen als unter den computerspielsüchtigen Jugendlichen.

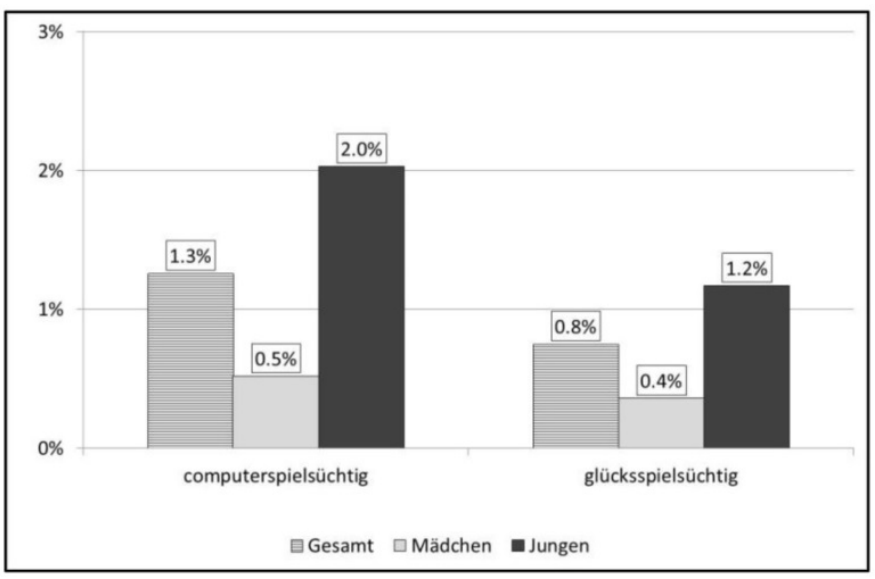

Abbildung 2. Anteil von glücksspiel- und computerspielsüchtigen Jungen und Mädchen. 
Es besteht eine leichte Überlappung zwischen beiden Störungen, indem 5 Jugendliche (4 Jungen und 1 Mädchen) sowohl die Kriterien einer Computerspiel- als auch einer Glücksspielsucht erfüllen. Dies bedeutet, dass die computerspielsüchtigen Jugendlichen zu 9.6 Prozent auch als glücksspielsüchtig und die glücksspielsüchtigen Jugendliche zu 15.2 Prozent auch als computerspielsüchtig eingestuft werden. Es findet sich somit eine relevante Komorbidität zwischen beiden Problemverhaltensweisen.

Im nächsten Schritt soll analysiert werden, ob sich glücksspielsüchtige und computerspielsüchtige Jugendliche in Hinblick auf objektive Belastungsindikatoren unterscheiden und wie stark die Belastung gegenüber einer Referenzgruppe unauffälliger Jugendlicher ausfällt. Dabei werden Variablen gesundheitlicher und funktionsbezogener Belastung einbezogen. Die fünf Jugendlichen, die sowohl als computerspiel- als auch als glücksspielsüchtig klassifiziert wurden, werden aus den Analysen ausgeschlossen. Ferner wird die Auswertung auf die von beiden Störungen in besonderer Weise betroffenen Jungen beschränkt, damit ein konfundierender Einfluss des Geschlechtes ausgeschlossen werden kann. Als Kovariate wird das Alter kontrolliert, womit die Gruppenmittelwerte um den Einfluss des Alters bereinigt werden (vgl. Tabelle 2).

Im Hinblick auf gesundheitsbezogene Beeinträchtigungsindikatoren weisen sowohl computerspiel- als auch glücksspielsüchtige Jungen eine verminderte Schlafzeit auf. Computerspielsüchtige Jungen schlafen rund 67 Minuten und glücksspielsüchtige Jungen 75 Minuten weniger als unauffällige Jungen. Zudem weisen in beiden Störungsgruppen 20 Prozent der Jungen eine aktuelle Schlafstörung auf, berichten also darüber, in der letzten Woche immer Probleme mit dem Einschlafen gehabt zu haben, während dies nur auf 2 Prozent der unauffälligen Jungen zutrifft. Hinsichtlich der Maße zum psychischen und körperlichen Wohlbefinden sowie Selbstvertrauen erscheinen beide Gruppen hingegen eher unauffällig. Nur bei den glücksspielsüchtigen Jugendlichen zeigt sich ein noch knapp signifikant vermindertes körperliches Wohlbefinden. Beide Gruppen weisen jedoch in signifikant höherem Maße Suizidgedanken auf, wobei die glücksspielsüchtigen Jungen hiervon noch deutlich stärker betroffen sind als die computerspielsüchtigen Jungen. In Hinblick auf die Suizidalität liegt eine besonders hohe Effektstärke vor die darauf hindeutet, dass dieses Merkmal besonders stark zwischen den Gruppen variiert. 
Tabelle 2. Vergleich unauffälliger, computerspielsüchtiger und glücksspielsüchtiger Jungen.

\begin{tabular}{|c|c|c|c|c|c|}
\hline Variablen & $\begin{array}{c}\text { Unauffällig } \\
\mathrm{n}=1.763 \\
M(S E)\end{array}$ & $\begin{array}{c}\text { VGA } \\
\mathrm{n}=36 \\
M(S E)\end{array}$ & $\begin{array}{c}\mathrm{GS} \\
\mathrm{n}=\mathbf{2 0} \\
M(S E)\end{array}$ & $\begin{array}{c}\text { VGA } \\
\text { vs. GS }\end{array}$ & Statistik \\
\hline \multicolumn{6}{|l|}{ Gesundheit } \\
\hline Schlafzeit (h/min) & $8: 13(0: 02)$ & $\begin{array}{c}7: 06 \\
(0: 13)^{* * *}\end{array}$ & $\begin{array}{c}6: 58 \\
(0: 17) * * *\end{array}$ & $\begin{array}{l}\mathrm{VGA}= \\
\mathrm{GS}\end{array}$ & $\begin{array}{l}F(2,1760)=23.63 \\
p<.001, \eta_{p}{ }^{2}=.026\end{array}$ \\
\hline $\begin{array}{l}\text { Aktuelle Schlafstö- } \\
\text { rung }\end{array}$ & $.02(.00)$ & $\begin{array}{c}.20 \\
(.03)^{* * *}\end{array}$ & $\begin{array}{c}.20 \\
(.04)^{* * *}\end{array}$ & $\begin{array}{l}\text { VGA }= \\
\text { GS }\end{array}$ & $\begin{array}{l}F(2,1758)=30.66 \\
p<.001, \eta_{p}{ }^{2}=.034\end{array}$ \\
\hline $\begin{array}{l}\text { Körperliches } \\
\text { Wohlbefinden }\end{array}$ & $16.0(.06)$ & $\begin{array}{l}15.4 \\
(.43)^{\mathrm{ns}}\end{array}$ & $\begin{array}{l}14.3 \\
(.59)^{*}\end{array}$ & $\begin{array}{l}\text { VGA }= \\
\text { GS }\end{array}$ & $\begin{array}{l}F(2,1735)=4.85 \\
p<.01, \eta_{p}{ }^{2}=.006\end{array}$ \\
\hline $\begin{array}{l}\text { Psychisches Wohl- } \\
\text { befinden }\end{array}$ & $16.4(.05)$ & $\begin{array}{l}15.5 \\
(.38)^{\mathrm{ns}}\end{array}$ & $\begin{array}{l}15.6 \\
(.50)^{\mathrm{ns}}\end{array}$ & $\begin{array}{l}\mathrm{VGA}= \\
\mathrm{GS}\end{array}$ & $\begin{array}{l}F(2,1742)=4.03, \\
p<.05, \eta_{p}{ }^{2}=.005\end{array}$ \\
\hline Selbstvertrauen & $14.2(.07)$ & $\underset{\mathrm{ns}}{13.1(.48)}$ & $\begin{array}{l}13.1 \\
(.64)^{\mathrm{ns}}\end{array}$ & $\begin{array}{l}\mathrm{VGA}= \\
\mathrm{GS}\end{array}$ & $\begin{array}{l}F(2,1696)=4.02 \\
p<.05, \eta_{p}{ }^{2}=.005\end{array}$ \\
\hline $\begin{array}{l}\text { Häufige Suizidge- } \\
\text { danken }\end{array}$ & $.02(.00)$ & $\begin{array}{c}.11 \\
(.03)^{* * *}\end{array}$ & $\begin{array}{c}.53 \\
(.04)^{* * *}\end{array}$ & $\begin{array}{l}\text { VGA }< \\
\text { GS*** }\end{array}$ & $\begin{array}{l}F(2,1730)=100.54 \\
p<.001, \eta_{p}{ }^{2}=.104\end{array}$ \\
\hline \multicolumn{6}{|l|}{ Funktionsniveau } \\
\hline $\begin{array}{l}\text { Funktionsniveau } \\
\text { im Alltag }\end{array}$ & $14.3(.06)$ & $\begin{array}{c}12.7 \\
(.43)^{* * *}\end{array}$ & $\begin{array}{c}11.9 \\
(.61)^{* * *}\end{array}$ & $\begin{array}{l}\text { VGA = } \\
\text { GS }\end{array}$ & $\begin{array}{l}F(2,1704)=13.26 \\
p<.001, \eta_{p}{ }^{2}=.015\end{array}$ \\
\hline $\begin{array}{l}\text { Notendurchschnitt } \\
\text { (letztes Zeugnis) }\end{array}$ & $3.0(.02)$ & $\begin{array}{c}3.4 \\
(.12)^{* *}\end{array}$ & $\begin{array}{c}3.1 \\
(.16)^{\mathrm{ns}}\end{array}$ & $\begin{array}{l}\text { VGA }= \\
\text { GS }\end{array}$ & $\begin{array}{l}F(2,1672)=5.85 \\
p<.01, \eta_{p}{ }^{2}=.007\end{array}$ \\
\hline $\begin{array}{l}\text { Geschwänzte } \\
\text { Schulstunden (letz- } \\
\text { tes HJ) }\end{array}$ & $3.7(.35)$ & $\begin{array}{c}8.2 \\
(2.42)^{\mathrm{ns}}\end{array}$ & $\begin{array}{c}16.3 \\
(3.25)^{* * *}\end{array}$ & $\begin{array}{l}\text { VGA = } \\
\text { GS }\end{array}$ & $\begin{array}{l}F(2,1765)=9.01 \\
p<.001, \eta_{p}^{2}=.010\end{array}$ \\
\hline
\end{tabular}

Anmerkung. VGA = Computerspielsüchtige. $\mathrm{GS}=$ Glücksspielsüchtige. Unauffällig = im Hinblick auf Computerspiel- und Glücksspielverhalten als unauffällig klassifiziert (CSASII $<35$ \& DSM-IV-MR-J < 4). Computerspielabhängigkeit wurde klassifiziert bei CSASII $>=42$. Glücksspielsucht wurde klassifiziert bei DSM-IV-MR-J $>=4$. Schlafzeit wurde erfasst als die Differenz zwischen dem Zubettgehen am Vorabend der Befragung und dem morgendlichen Aufstehen am Tag der Befragung. Eine Schlafstörung wurde angenommen, wenn Jugendliche für die letzte Woche berichten, immer Schwierigkeiten mit dem Einschlafen gehabt zu haben. Messung von Körperlichem Wohlbefinden, Psychischem Wohlbefinden, Funktionsniveau im Alltag und Selbstvertrauen mit den gleichnamigen Unterskalen des Kiddo-KINDL ${ }^{\mathrm{R}}$ (Ravens-Sieberer \& Bullinger, 2000). Häufige Suizidgedanken wurden angenommen, wenn Jugendliche auf das Item „Ich denke immer wieder an den Tod oder daran, mich umzubringen“ der Depressionsskala des DISYPS-II (Döpfner, Görtz-Dorten \& Lehmkuhl, 2008) mit „weitgehend“ oder „besonders“ geantwortet haben. Notendurchschnitt basierend auf den Angaben der Jugendlichen zu den Noten im letzten Zeugnis in den Fächern Deutsch, Mathematik, Englisch und Biologie. Geschwänzte Schulstunden im letzten Halbjahr basierend auf der Anzahl geschwänzter Einzelstunden und ganzer Schultage und Verrechnung in einem Index (Schultage x $5+$ Einzelstunden). Signifikanztest mit ANCOVA. Alle Mittelwerte wurden um den Einfluss der Kovariate Alter bereinigt. Post hoc Vergleiche mit Bonferroni Korrektur. In den Spalten VGA und GS wird die Abweichung von den unauffälligen Jungen gegeben. In der Spalte VGA vs. GS wird die Abweichung zwischen beiden Störungen angegeben. ${ }^{*} p<.05 ;{ }^{*} p<.01 ; * * * p<.001$; ns $=$ nicht signifikant. 
Im Hinblick auf funktionelle Beeinträchtigungsindikatoren weisen sowohl computerspiel- als auch glücksspielsüchtige Jugendliche ein vermindertes Funktionsniveau im Alltag auf. Dies bedeutet, dass die betroffenen Jugendlichen stärker das Gefühl haben, die Aufgaben in der Schule nicht gut zu schaffen, sich weniger für den Unterricht zu interessieren, sich Sorgen um ihre Zukunft zu machen und Angst vor schlechten Noten zu haben.

Beide Gruppen fallen zusätzlich hinsichtlich schulischer Leistungsparameter auf, indem computerspielsüchtige Jungen einen signifikant verminderten Notendurchschnitt und glücksspielsüchtige Jungen ein signifikant erhöhtes Schulschwänzen aufweisen.

Der Posthoc-Test auf Gruppenunterschiede erbringt in weiten Teilen keine bedeutsamen Unterschiede zwischen computerspiel- und glücksspielsüchtigen Jungen. Von Suizidgedanken sind glücksspielsüchtige Jungen allerdings deutlich häufiger betroffen.

\section{Diskussion}

Eine Besonderheit der vorgelegten Auswertungen besteht darin, dass stoffgebundene und stoffungebundene Konsummuster methodisch vergleichbar erfasst wurden, und damit ihre Relevanz für Jungen und Mädchen systematisch miteinander verglichen werden konnte. Dabei konnte gezeigt werden, dass Jungen - wie anhand der vorhandenen Datenlage zu erwarten - mit Ausnahme des Zigarettenkonsums von allen problematischen Konsummustern häufiger betroffen sind als Mädchen. Dies konnte insbesondere in Hinblick auf das Computerspiel- und Glücksspielverhalten gezeigt werden. Beide Verhaltensweisen werden häufiger von Jungen in problematischer und in pathologischer Weise betrieben als von Mädchen.

Insgesamt fallen die Belastungswerte der im Jahr 2011 erhobenen ADIGD-Daten für problematischen Substanzkonsum in der Subgruppe der Neuntklässler deutlich geringer aus als in den im Jahr 2007 erhobenen deutschlandrepräsentativen Neuntklässlerdaten (Baier \& Rabold, 2009). Dies kann auf verschiedene mögliche Gründe zurückgeführt werden: Zum einen ist darauf hinzuweisen, dass in der vorliegenden (nicht repräsentativen) Studie ein höherer Anteil an Gymnasiasten befragt wurde als in der Neuntklässlerbefragung 2007 (41\% vs. 30\%). Gerade bei den Gymnasiasten handelt es sich jedoch um eine Gruppe, die insgesamt einen weitaus geringeren problematischen Konsum aufweist als alle anderen Schülerpo- 
pulationen. Weiterhin ist der Migrantenanteil in der vorliegenden Stichprobe etwas höher ausgeprägt als in der bundesweiten Neuntklässlerbefragung, was auf die regionalen Besonderheiten im Raum Hannover zurückzuführen ist (32\% vs. 27\%). Gerade unter türkischstämmigen Jugendlichen besteht jedoch ein weitaus geringerer Alkoholkonsum als unter deutschstämmigen Jugendlichen (Baier \& Rabold, 2009). Neben diesen Besonderheiten der vorliegenden regional geprägten Stichprobe ist jedoch auch ein allgemeiner Rückgang des problematischen Drogenkonsums in den letzten Jahren als ursächlich für die geringeren Belastungszahlen anzunehmen. Jedoch ist anzumerken, dass dieser Rückgang im Vergleich beider genannten Datenquellen aus den genannten Gründen sicherlich überschätzt wird.

\section{Literatur}

Achab, S., Nicolier, M., Mauny, F., Monnin, J., Trojak, B., Vandel, P. S. et al. (2011). Massively multiplayer online role-playing games: comparing characteristics of addic vs. non-addict online recruited gamers in a French adult population. BMC Psychiatry, 11(8), 144-154.

American Psychiatric Association. (2013). Diagnostic and Statistical Manual of Mental Disorders, Fifth Version $\left(D S M-5^{t m}\right)$. Arlington: American Psychiatric Publishing.

Anderson, P., Moller, L. \& Galea, G. (2012). Alcohol in the European Union: Consumption, harm, and policy approaches: World Health Organization.

Baier, D. \& Rabold, S. (2009). Drogenkonsum im Jugendalter: Verbreitung, Bedingungsfaktoren und Zusammenhang mit Gewaltverhalten. Zeitschrift für Jugendkriminalrecht und Jugendhilfe, 4, 292-305.

Baier, D., Rabold, S., Kappes, C. \& Kudlacek, D. (2009). Sicherheit und Kriminalität in Stade. Ergebnisse einer Schüler und Erwachsenen Befragung. (Forschungsbericht No. 106). Hannover: Kriminologisches Forschungsinstitut Niedersachsen.

Batthyány, D., Müller, K. W., Benker, F. \& Wölfling, K. (2009). Computerspielverhalten: Klinische Merkmale von Abhängigkeit und Missbrauch bei Jugendlichen. Wiener Klinische Wochenschrift, 121(15), 502-509.

Becker, J. B. \& Hu, M. (2008). Sex differences in drug abuse. Front Neuroendocrinol, 29(1), 36-47.

Beutel, M. E., Hoch, C., Wölfling, K. \& Müller, K. W. (2011). Klinische Merkmale der Computerspiel- und Internetsucht am Beispiel der Inanspruchnehmer einer Spielsuchtambulanz. Zeitschrift für Psychosomatische Medizin und Psychotherapie, 57, 77-90. 
Bioulac, S., Arfi, L. \& Bouvard, M. P. (2008). Attention deficit/ hyperactivity disorder and video games: a comparative study of hyperactive and control children. European Psychiatry, 23(2), 134-141.

Bundeszentrale für gesundheitliche Aufklärung. (2012). Die Drogenaffinität Jugendlicher in der Bundesrepublik Deutschland 2011. Der Konsum von Alkohol, Tabak und illegalen Drogen: aktuelle Verbreitung und Trends. Köln: Bundeszentrale für gesundheitliche Aufklärung.

Delfabbro, P. H., Winefield, A. H. \& Anderson, S. (2009). Once a gambler - always a gambler? A longitudinal analysis of gambling patterns in young people making the transition from adolescence to adulthood. International Gambling Studies 9(2), 151-163.

Die Drogenbeauftragte der Bundesregierung. (2012). Drogen- und Suchtbericht: Bundesministerium für Gesundheit.

Fend, H. (2005). Rauchen als Risiko-Indikator für jugendliche Lebensstile. Stabilität und Folgen für soziale Übergänge ins Erwachsenalter. Zeitschrift für Soziologie und Erziehung und Sozialisation, 25(1), 82-94.

Festl, R., Scharkow, M. \& Quandt, T. (2013). Problematic computer game use among adolescents, younger and older adults. Addiction, 108, 592-599.

Fisher, S. (1999). A prevalence study of gambling and problem gambling in British adolescents. Addiction Research, 7(6), 509-538.

Fisher, S. (2000). Developing the DSM-IV-DSM-IV criteria to identify adolescent problem gambling in non-clinical populations. Journal of Gambling Studies, $16(2 / 3), 253-273$.

Gentile, D. A. (2009). Pathological Video-Game Use Among Youth Ages 8 to 18: A National Study. Psychological Science, 20(5), 594-602.

Gentile, D. A., Choo, H., Liau, A., Sim, T., Li, D., Fung, D. et al. (2011). Pathological Video Game Use Among Youths: A Two-Year Longi-tudinal Study. Pediatrics, 127(2), 319-329.

Ihle, W. \& Esser, G. (2002). Epidemiologie psychischer Störungen im Kindes- und Jugendalter: Prävalenz, Verlauf, Komorbidität und Geschlechterunterschiede. Psychologische Rundschau, 53(4), 159-169.

Kraus, L., Pabst, A. \& Müller, S. (2011). Epidemiologischer Suchtsurvey 2009 Repräsentativerhebung zum Gebrauch und Missbrauch psychoaktiver Substanzen bei Erwachsenen in Hamburg. München: IFT Institut für Therapieforschung.

Lee, S. Y. \& Kwon, J. H. (2001). Impulsivity, social problem-solving abilities, and communication style of adolescent internet game addicts The Korean Journal of Clinical Psychology 20, 67-80.

Mentzoni, R. A., Brunborg, G. S., Molde, H., Myrseth, H., Skouverøe, K. J. M., Hetland, J. et al. (2011). Problematic Video Game Use: Estimated Prevalence and Associations with Mental and Physical Health. Cyberpsychology, Behavior and Social Networking, 14(10), 591-596. 
Meyer, C., Rumpf, H.-J., Kreuzer, A., de Brito, S., Glorius, S., Jeske, C. et al. (2011). Pathologisches Glücksspielen und Epidemiologie (PAGE): Entstehung, Komorbidität, Remission und Behandlung. Endbericht an das Hessische Ministerium des Innern und für Sport. Greifswald/ Lübeck: Universitäten Greifswald und Lübeck.

Meyer, G. \& Bachmann, M. (2011). Spielsucht: Ursachen, Therapie und Prävention von glücksspielbezogenem Suchtverhalten (3. Auflage). Heidelberg: Springer Verlag.

Mößle, T. (2012). dick, dumm, abhängig, gewalttätig? Problematische Mediennutzungsmuster und ihre Folgen im Kindesalter. Ergebnisse des Berliner Längsschnitt Medien. Baden-Baden: Nomos Verlag.

Mößle, T. \& Rehbein, F. (2013). Predictors of problematic video game usage in childhood and adolescence. Sucht, 59(3), 129-142.

Muthén, B. O. \& Muthén, L. K. (2000). The development of heavy drinking and alcohol-related problems from age 18 to 37 in a U.S. national sample. Journal of Studies on Alcohol and Drugs, 61, 290-300.

Pabst, A., Piontek, D., Kraus, L. \& Müller, S. (2010). Substanzkonsum und substanzbezogene Störungen. Ergebnisse des Epidemiologischen Suchtsurveys 2009. Sucht, 56(5), 327-336.

Pinquart, M. \& Silbereisen, R. K. (2002). Gesundheitsverhalten im Kindes- und Jugendalter Bundesgesundheitsblatt - Gesundheitsforschung - Gesundheitsschutz, 45, 873-878.

Rehbein, F., Kleimann, M. \& Mößle, T. (2009). Computerspielabhängigkeit im Kindes- und Jugendalter. Empirische Befunde zu Ursachen, Diagnostik und Komorbiditäten unter besonderer Berücksichtigung spielimmanenter Abhängigkeitsmerkmale (Forschungsbericht No. 108). Hannover: Kriminologisches Forschungsinstitut Niedersachsen.

Rehbein, F., Kleimann, M. \& Mößle, T. (2010). Prevalence and Risk Factors of Video Game Dependency in Adolescence: Results of a German Nationwide Survey. Cyberpsychology, Behavior, and Social Networking, 13(3), 269-277.

Rehbein, F. \& Mößle, T. (2013). Video game addiction and Internet addiction: Is there a need for differentiation? Sucht, 59(3), 153-164.

Rehbein, F., Mößle, T., Jukschat, N. \& Zenses, E.-M. (2011). Zur psychosozialen Belastung exzessiver und abhängiger Computerspieler im Jugend- und Erwachsenenalter. Suchttherapie, 12(2), 64-71.

Schmidt, J.-H., Drosselmeier, M., Rohde, W. \& Fritz, J. (2011). Problematische Nutzung und Abhängigkeit von Computerspielen. In J. Fritz, C. Lampert, J.-H. Schmidt \& T. Witting (Hrsg.), Kompetenzen und exzessive Nutzung bei Computerspielern: Gefordert, gefördert, gefährdet (61 ed.). Berlin: Vistas Verlag.

Schmidt, L. \& Kähnert, H. (2003). Konsum von Glücksspielen bei Kindern und Jugendlichen: Verbreitung und Konsum. Abschlussbericht an das Ministerium für Gesundheit, Soziales, Frauen und Familie des Landes Nordrhein-Westfalen. Bielefeld: Universität Bielefeld, Fakultät für Gesundheitswissenschaften. 
Starcevic, V., Berle, D., Porter, G. \& Fenech, P. (2011). Problem Video Game Use and Dimensions of Psychopathology. International Journal of Mental Health and Addiction, 9(3), 248-256.

World Health Organization. (2009). Global health risks: Mortality and burden of disease attributable to selected major risks. Geneva: World Health Organization.

Young, K. S. (2010). A therapist's guide to assess and treat Internet addiction. Psychiatry Research, 1-45. 